\title{
PERSPECTIVAS SOBRE ESTÁNDARES CURRICULA- RES PARA EDUCACIÓN FÍSICA EN MÉXICO
}

\section{PERSPECTIVES ON CURRICULAR STANDARDS FOR PHYSICAL EDUCATION IN MEXICO}

\section{Dr. Salvador Jesús López Alonzo ${ }^{*}$, Dr. Julio Cesar Guedea Delgado², Dr. Oscar Núñez Enríquez ${ }^{3}$ Dra. Ma Concepción Soto Valenzuela ${ }^{4}$, Dr. Gabriel Gastélum Cuadras ${ }^{5}$}

Universidad Autónoma de Chihuahua, Chihuahua, México

1Email: salopez@uach.mx (iD https://orcid.org/0000-0002-9637-9179

2. Email: jcguedea@uach.mx (iD) https://orcid.org/0000-0001-5588-1130

3. Email: onuneze@uach.mx (iD https://orcid.org/0000-0001-6573-6762

4. Email: masoto@uach.mx (iD) https://orcid.org/0000-0002-1043-7631

5. Email: gastelum@uach.mx (iD https://orcid.org/0000-0002-8931-1125

Como Citar: López Alonzo, S. J., Guedea Delgado, J. C. ., Núñez Enríquez, O. ., Soto Valenzuela , M. C. ., \& Gastélum Cuadras , G. . (2021). Perspectivas sobre Estándares Curriculares para Educación Física en México. Revista Publicando, 8(28), 99-109. https://doi.org/10.51528/rp.vol8. id 2152

\begin{abstract}
RESUMEN:
Objetivo: Analizar las perspectivas de grupos focales sobre el uso de estándares curriculares para la Educación Física en la enseñanza básica. Método: Participaron docentes de educación básica $(n=3)$, Educación Física $(n=3)$ y educación superior $(n=3)$, se seleccionaron tres estándares curriculares para la Educación Física extraídos de una revisión documental para el análisis temático y se generaron diferentes categorías. Resultados: Principales categorías; 1 ) formación profesional del educador físico, 2) capacitación y certificación a los docentes en servicio, 3) aprendizajes esperados, estrategias didácticas y criterios de evaluación, 4) calidad de la Educación Física. Conclusiones: El trabajo con estándares curriculares puede ser una oportunidad para mejorar la Educación Física, sin embargo, se requiere de un esfuerzo conjunto entre los diferentes sectores educativos y de la sociedad. Además, es necesario crear políticas educativas y de salud pública para fortalecer esta asignatura, así como la formación de los educadores físicos.
\end{abstract}

\section{PALABRAS CLAVE:}

Estándares, Educación Física, Currículo, Grupos Focales, Educación Básica.

\section{ABSRTACT:}

Objective: To analyze the perspectives of focus groups on the use of curricular standards for physical education in basic education. Method: Teachers of basic education $(n=3)$, physical education $(n=3)$ and higher education $(n=3)$ participated, three curricular standards for physical education were selected, extracted from a documentary review for thematic analysis and generated different categories. Results: Main categories; 1) professional training of the physical educator, 2) training and certifying in-service teachers, 3) expected learning, didactic strategies and evaluation criteria, 4) quality of physical education. Conclusions: Functioning with curricular standards can be an opportunity to improve physical education; however, a joint effort is required between different educational sectors and society. In addition, it is necessary to create educational and public health policies to strengthen this subject and the training of physical educators.

\section{KEYWORDS:}

Standards, Physical Education, Curriculum, Focus Groups, Basic Education. 


\section{INTRODUCCIÓN}

A ctualmente la Educación Física es una asignatura clave en el currículo académico de educación básica para mejorar y conservar la salud, desde un plano de actividad física y deporte escolar, sin embargo, aún existen inconsistencias en los planes curriculares en México y algunos países de Latino América (García y Del Basto, 2017; Gallo y Urrego, 2015; Reyes, Rivas y Pávez-Adasme, 2020). Han pasado más de 15 años desde que la Educación Física ha sufrido cambios en el campo disciplinar, el cual involucra una necesidad de trabajar desde una postura epistemológica que conlleva la práctica de múltiples disciplinas (Melnychuk, Robinson, Lu, Chorney y Randall, 2011). La Anatomía, Fisiología, Fisiología del ejercicio, Biomecánica, Kinesiología, Sociología y Psicología del deporte, se han convertido en disciplinas fundamentales en los procesos de enseñanza y aprendizaje de la Educación Física (Melnychuk et al., 2011). Los cambios en el área disciplinar y currículo de estas asignaturas, son recomendaciones y lineamientos desarrollados por el ICHPER SD (International Council for Health, Physical Education, Recreation, Sport, and Dance), los cuales buscan mejorar la calidad en el servicio de esta asignatura. En la actualidad la Organización de las Naciones Unidas para la Educación, la Ciencia y la Cultura (UNESCO), concibe a la Educación Física de calidad como: "la experiencia de aprendizaje planificada, progresiva e inclusiva que forma parte del currículo en educación infantil, primaria y secundaria”. Es necesario que se comprenda que la asignatura de Educación Física es la única que promueve académicamente un compromiso con la actividad física y deporte a lo largo de la vida, además de ser considerada parte elemental del currículo académico (UNESCO, 2015). Según el SHAPE-AMERICA (America-Society of Health and Physical Educators) la Educación Física contribuye a desarrollar experiencias apropiada de aprendizajes en los escolares para ayudarles a adquirir las habilidades psicomotrices, locomotrices, mejorar la condición física, la salud, comprensión cognitiva y aptitudes sociales que necesitan para llevar una vida físicamente activa y saludable a lo largo de la vida (UNESCO, 2015; SHAPE-AMERICA, 2015). En México la Encuesta Nacional de Salud y Nutrición de Medio Camino 2016, muestra resultados alarmantes en los escolares entre 5 y 11 años, con un $33.2 \%$ de sobrepeso y obesidad, lo que es considerado como una problemática de salud pública. Si se mejora la calidad en el servicio de la Educación Física, esta puede ser de gran relevancia para poder combatir el sobrepeso y la obesidad infantil en la educación básica, pero, es necesaria una Educación Física de calidad, con políticas claras y pertinentes que realmente pueda ayudar a disminuir estos porcentajes tan alarmantes de sobrepeso y obesidad (UNESCO, 2015) y contribuir a una educación básica integral.

\section{ESTÁNDARES CURRICULARES}

Los estándares curriculares forman parte de una política educativa de calidad, estos estándares curriculares se han desarrollado en varias organizaciones y para PISA (Programme for International Student Assessment, 2006) forman parte del modelo educativo global, contribuyen a establecer y definir esencialmente los contenidos y los aprendizajes que deben adquirir los alumnos (ICHPER SD, 2009; PISA, 2006). En relación con la Educación Física los estándares curriculares muestran de forma global la naturaleza del currículo, sin dejar a un lado el hecho de que se debe tomar en cuenta el contexto, la región o el país (ICHPER SD, 2009; Liang, Walls, y Lu., 2005). Los estándares curriculares pueden contribuir a desarrollar una postura más congruente con lo recomendado por la UNESCO para esta asignatura, estos estándares pueden resolver las críticas que se han hecho en diferentes países de Latino América, por la falta de congruencia en los contenidos e implementación de la Educación Física (Marani, Sanches y dos Santos, 2017; UNESCO, 2015). En México no existen estándares curriculares para la Educación Física, según lo muestran los planes y programas 2011, así como la propuesta educativa del 2016 de la SEP (Secretaría de Educación Pública), por lo que sería relevante implementarlos en la educación básica para mejorar la calidad de esta asignatura.

En general los estándares curriculares se refieren a lo que el estudiante debe saber y poder hacer como resultado del plan de estudio, el cual es clave para elevar la calidad en la educación, por ello, la Educación Física, en su calidad de asignatura para la vida, debe contemplarlos, buscando mejorar la calidad de la misma (ICHPER SD, 2009; PISA, 2006; SHAPE-AMERICA, 2015). Los estándares curriculares en Educación Física establecen de manera implícita definiciones operativas que permiten las distinciones y relaciones entre esta asignatura y los demás campos afines como la danza, actividad física, salud, recreación y deporte. Estos estándares fijan criterios para evaluar los aprendizajes de los escolares según sea la edad o el periodo escolar, permiten el diálogo global, la investigación, la comprensión, así como los intercambios conceptuales, procedimentales y actitudinales entre los profesionales de esta asignatura y organizaciones especialistas en la materia (ICHPER•SD, 2009; SHAPE-AMERICA, 2015).

Los estándares curriculares que se analizan en este estudio son seleccionados por ser los más comunes y son basados en diferentes organizaciones especializadas en Educación Física, como el ICHPER•SD o el SHAPE AMERICA (Ver Tabla 1). Para contextualizar los estándares curricula- 
직

res, se puede entender que el currículum incluye todos los conocimientos, habilidades y experiencias de aprendizaje que se ofrecen a los estudiantes dentro de un programa escolar y los estándares curriculares son lo que se espera del

Tabla 1. Estándares curriculares consultados para la Educación Física a nivel internacional en educación básica

\begin{tabular}{|c|c|c|}
\hline CHINA/ASIA-CME & ICHPER•SD & EUA-SHAPE AMERICA \\
\hline $\begin{array}{l}\text { Es físicamente saludable } \\
\text { y demuestra competencia } \\
\text { en diferentes formas de } \\
\text { movimientos para adquirir } \\
\text { conocimientos y habil- } \\
\text { idades para la actividad } \\
\text { física y la salud. }\end{array}$ & $\begin{array}{l}\text { Demuestra compe- } \\
\text { tencia en una gran } \\
\text { variedad y formas de } \\
\text { movimiento. }\end{array}$ & $\begin{array}{l}\text { El individuo físicamente alfa- } \\
\text { betizado. } \\
\text { Demuestra competencias en una } \\
\text { variedad de habili-dades moto- } \\
\text { ras y patrones de movimiento }\end{array}$ \\
\hline $\begin{array}{l}\text { Participa regularmente en } \\
\text { actividades físicas }\end{array}$ & $\begin{array}{l}\text { Aplica conceptos } \\
\text { y principios de } \\
\text { movimiento, para el } \\
\text { aprendizaje de las ha- } \\
\text { bilida-des motoras. }\end{array}$ & $\begin{array}{l}\text { Aplica el conocimiento de con- } \\
\text { ceptos, principios, es-trategias } \\
\text { y tácticas rela-cionadas con } \\
\text { el movimiento y rendimiento } \\
\text { físi-co. }\end{array}$ \\
\hline $\begin{array}{l}\text { Demuestra comunicación } \\
\text { y participación con otros } \\
\text { partícipes en las diferentes } \\
\text { actividades físicas. }\end{array}$ & $\begin{array}{l}\text { Logra y mantiene } \\
\text { una mejora en la } \\
\text { condición física y la } \\
\text { salud. }\end{array}$ & $\begin{array}{l}\text { Demuestra conocimientos y } \\
\text { destrezas para lograr y mantener } \\
\text { un nivel saludable de actividad } \\
\text { física y condición física. }\end{array}$ \\
\hline $\begin{array}{l}\text { Demuestra responsabili- } \\
\text { dad en la salud individual } \\
\text { y con la sociedad, así como } \\
\text { estilos de vida saludable. }\end{array}$ & $\begin{array}{l}\text { Demuestra un estilo } \\
\text { de vida activo. }\end{array}$ & $\begin{array}{l}\text { Demuestra respeto personal y } \\
\text { social con él mismo y los demás. }\end{array}$ \\
\hline \multirow[t]{3}{*}{$\begin{array}{l}\text { Demuestra una actitud } \\
\text { positiva hacia la vida y a } \\
\text { las actividades físicas. }\end{array}$} & $\begin{array}{l}\text { Demuestra un } \\
\text { comportamiento } \\
\text { de responsabilidad } \\
\text { personal y social en } \\
\text { escenarios de activi- } \\
\text { dad física. }\end{array}$ & \multirow[t]{3}{*}{$\begin{array}{l}\text { Reconoce el valor de la actividad } \\
\text { física para la salud, el disfrute, } \\
\text { el desafío, la autoexpresión e } \\
\text { interacción social. }\end{array}$} \\
\hline & $\begin{array}{l}\text { Demuestra compren- } \\
\text { sión y respeto por las } \\
\text { diferencias entre la } \\
\text { gente en escenarios } \\
\text { de actividad física. }\end{array}$ & \\
\hline & $\begin{array}{l}\text { Comprende que las } \\
\text { actividades físicas } \\
\text { proveen de opor- } \\
\text { tunidades para el } \\
\text { disfrute, retos como } \\
\text { autoex-presión e } \\
\text { interacción social. }\end{array}$ & \\
\hline
\end{tabular}

Fuente: China regulation of physical education and student athletics (CME), 2001, International Council for Health, Physical Education, Recreation, Sport, and Dance (ICHPER•SD), 2009. SHAPE America -Society of Health and Physical Educators, 2015.

En la Figura. 1 puede observarse un ejemplo de la función operativa de un estándar curricular tomado del IHCPER SD con puntos de referencia y aprendizaje esperados desarrollados para la Educación Física en educación básica. Los estándares curriculares tienen una operatividad flexible, se pueden diseñar uno o varios puntos de referencia y desglosarse en aprendizajes esperados, estos pueden esclarecer la postura de esta asignatura. Además, los estándares son planeados para un periodo de tiempo prolongado de años. 


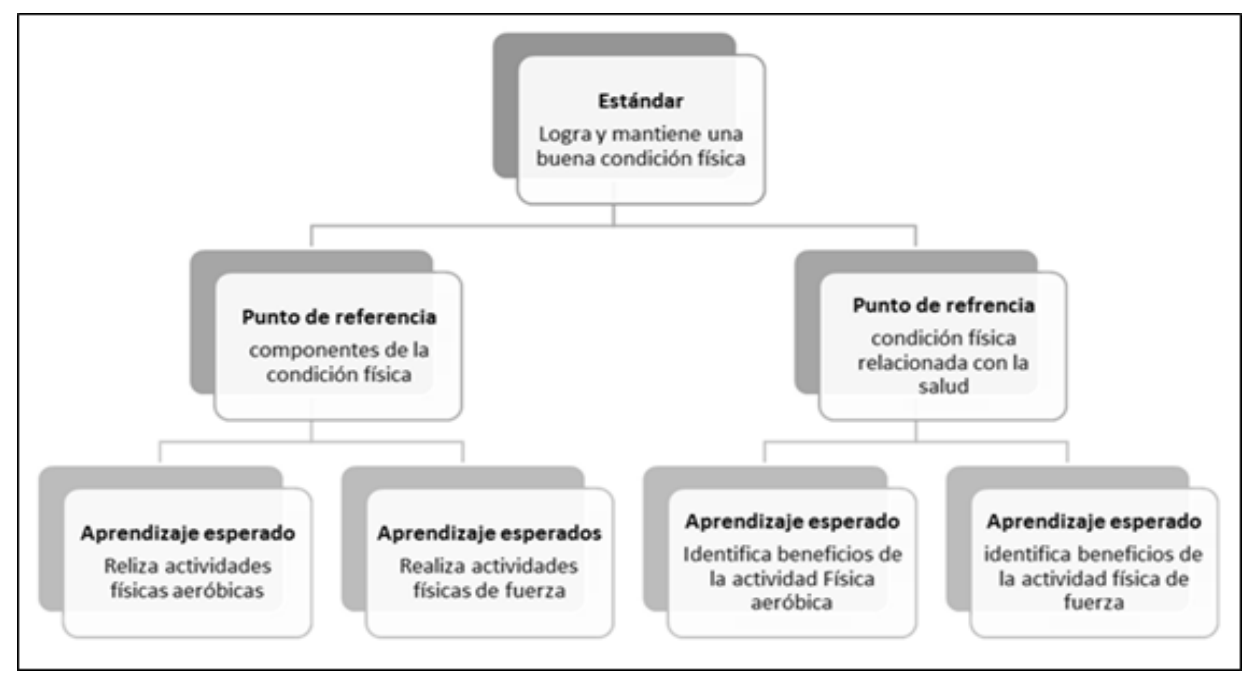

Figura 1. Función operativa de un estándar curricular basado en el ICHPER SD,

estudiante (Lound y Tannehill, 2014).

En esta investigación se pretende desarrollar ideas y perspectivas de los Grupos Focales (GF), sobre el uso de los estándares curriculares para la Educación Física en educación básica. Esta técnica investigativa se ha desarrollado como un método para recabar información colectivista, centrado en la experiencia y conocimiento de personajes clave relacionados con un tema en particular (Hamui-Sutton y Varela-Ruiz, 2013). En el presente estudio la temática y unidad de análisis, será los estándares curriculares para la Educación Física en educación básica, buscando identificar los beneficios y perjuicios al llevarlos a la práctica docente.

\section{METODOLOGIA}

\section{MUESTRA}

Participaron 9 sujetos en los GF, 6 mujeres y 3 hombres, seleccionados por conveniencia, el criterio de inclusión fue que tuvieran al menos cinco años de experiencia en docencia, en educación básica o educación superior. El promedio de edad fue de 42.1 años y una experiencia en la docencia promedio de 13.9 años. Se pudieron formar solo tres GF que aceptaron voluntariamente participar en este estudio; tres Asesores Técnicos Pedagógicos (ATP), los cuales desarrollan funciones de asesoramiento, evaluación y supervisión pedagógica a los docentes en servicio, pertenecientes a la SEP, tres Licenciados en Educación Física (LEF) al servicio de la SEP y tres Profesores Investigadores en Actividad Física (PIAF) pertenecientes a una Universidad Pública.

\section{INSTRUMENTOS}

Para el análisis temático se utilizaron tres estándares cu- rriculares esenciales y exclusivos para la Educación Física en educación básica, con base en la consulta hecha en internet, incluyendo a organizaciones especialistas en la temática (Ver Tablas 1 y 2.). Para la recopilación de las ideas y conceptos de los GF se desarrollaron los siguientes cuestionamientos:

¿Qué opina sobre los siguientes estándares curriculares planteados para la Educación Física en educación básica?

¿Según su opinión, cuál es la importancia de utilizar los siguientes estándares curriculares para la Educación Física en educación básica?

¿Cuáles serían las limitantes de utilizar estos estándares curriculares para la Educación Física en educación básica?

¿Cuáles serían las ventajas de utilizar estos estándares curriculares para la Educación Física en educación básica?

Tabla 2. Estándares curriculares para la Educación Física en educación básica como temática.

\section{Estándar El estudiante deberá:}

1. Demostrar dominio de habilidades motoras y formas de movimiento necesarios para participar en una gran variedad de actividades físicas.

2. Demostrar y aplicar el conocimiento de conceptos, principios y estrategias de movimien-to, necesarios para el aprendizaje de las habilidades motoras.

3. Evaluar los componentes de la condición física para mejorar el rendimiento físico y la salud.

Nota: Adaptado de estándares internacionales para la Educación Física en educación básica del ICHPER.SD (International Council for Health, Physical Education, Recreation, Sport and Dance, 2009) y del SHAPE America (America -Society of Health and Physical Educators, 2015). Ela- 
borado por autores.

\section{PROCEDIMIENTOS}

Los GF fueron seleccionados por conveniencia y tomando en cuenta la relación con la Educación Física, pedagogía y actividad física para la salud, las sesiones fueron organizadas por grupos en diferentes momentos, para los ATP se utilizó su recinto de trabajo, "la Mesa Técnica Única de Investigación Docente" perteneciente a la SEP y con sede en la ciudad de Chihuahua, México, se realizaron dos sesiones de sesenta minutos para no afectar actividades laborales, con permiso y ajustándose a los horarios establecidos por el jefe de departamento.

En el caso de los LEF y PIAF se planearon dos sesiones de sesenta minutos para comodidad y por disponibilidad de los grupos en diferentes días, las sesiones se realizaron en una universidad pública estatal en un horario establecido por los grupos. Para la recolección de datos de video grabaron las sesiones, posteriormente se analizó el discurso de los grupos tomando como Unidad de Análisis (UA) los estándares curriculares seleccionados y cuestionando a los GF sobres los mismos. Se realizó una transcripción escrita del discurso en Word, para analizar el discurso se desarrollaron diferentes categorías para cada grupo y estándar curricular.

\section{RESULTADOS}

En la Tabla 3 se muestran los rangos mínimos, máximos promedios y desviación estándar de la edad y experiencia docente en años de los GF que participaron en el estudio, se puede observar que el grupo con más experiencia profesional en docencia es el de los PIAF seguido de los ATP y LEF, respectivamente.

Tabla 3. Descriptivos de los GF por edad y años de experiencia.

\begin{tabular}{lllll}
\hline GF & $\begin{array}{l}\text { Rango } \\
\text { Mínimo }\end{array}$ & $\begin{array}{l}\text { Rango } \\
\text { Máximo }\end{array}$ & Media & D. etándar \\
\hline $\begin{array}{l}\text { ATP (n=3) } \\
\text { Edad (años) }\end{array}$ & 40 & 47 & 44.0 & 3.6 \\
$\begin{array}{l}\text { Experiencia } \\
\text { (años) }\end{array}$ & 12 & 15 & 13.3 & 1.5 \\
LEF (n=3) & & & & \\
Edad (años) & 32 & 40 & 35.7 & 4.0 \\
$\begin{array}{l}\text { Experiencia } \\
\text { (años) }\end{array}$ & 5 & 12 & 9.3 & 3.8 \\
$\begin{array}{l}\text { PIAF (n=3) } \\
\text { Edad (años) }\end{array}$ & 38 & 57 & 46.7 & 9.6 \\
$\begin{array}{l}\text { Experiencia } \\
\text { (años) }\end{array}$ & 12 & 30 & 19.0 & 9.6 \\
\hline
\end{tabular}

Nota: GF= Grupo Focal, ATP= Asesor Técnico Pedagógico, LEF= Licenciado en Educación Física, PIAF= Profesor Investigador en Actividad Física, D=desviación. Elaborado por autores.

Los ATP consideran que para llevar a cabo el estándar uno (Ver Tabla 4) es necesario desarrollar estrategias didácticas, aprendizajes esperados y criterios de evaluación. También se pueden observar ideas en las que, el trabajo con estándares curriculares podría ser difícil para el educador físico, debido a que no ha trabajado con ellos, sin embargo, conciben que podrían ser de importancia.

Tabla 4. Perspectivas de los ATP sobre el estándar curricular uno para Educación Física en educación básica.

Estándar curricular 1. Demuestra el dominio de habilidades motoras y formas de movimiento necesarios para participar en una gran variedad de actividades físicas.

Categorías

- Necesarios criterios de evaluación.

- Necesarias Estrategias didácticas.

- Es importante para que los estudiantes tengan la autoconfianza de participar en actividades físicas.

- Difícil el trabajo con estándares curriculares en Educación Física.

- Los profes no han trabajado con estándares.

- La propuesta de este estándar es muy interesante.

- Los profes de Educación Física sólo ponen juegos y deportes.

- La Educación Física es más que juegos y deportes.

- Es necesario desarrollar aprendizajes esperados para el estándar.

Nota: Elaborado por autores.

Las perspectivas de los ATP sobre el estándar dos (Ver Tabla 5) establecen que no se utilizan principios y conceptos en la clase de Educación Física y además solo se ponen juegos y deportes; este estándar podría darle formalidad académica a la clase de Educación Física.

Tabla 5. Perspectivas de los ATP sobre el estándar curricular dos para Educación Física en educación básica.

Estándar curricular 2. Demuestra y aplica conocimientos de conceptos, principios y estrategias de movimientos, necesarios para el aprendizaje de las habilidades motoras.

Categorías

- El profe nunca utiliza principios y conceptos.

- Solo ponen juegos y deportes.

- Sería importante para que esta materia pueda ser considerada realmente como académica y no de relleno.

- $\quad$ Es interesante que este estándar considere principios y conceptos en la Educación Física.

- La Educación Física no desarrolla muchos principios y conceptos.

- Solo ponen deportes, juegos y forman equipos.

- Podría contribuir a un conocimiento concreto sobre las habilidades motrices.

- $\quad$ Podría formalizar la Educación Física.

- Los profes no se preocupan por que los estudiantes desarrollen conocimientos de conceptos, principios o estrategias.

- En la política educativa no existen estándares curriculares.

Nota: Elaborado por autores. 
Los ATP (Ver Tabla 6), reconocen que no existen políticas sobre estándares curriculares para Educación Física en educación básica, el estándar 3 es considerado de importancia por la problemática de obesidad infantil, pero con una necesidad de crear estrategias didácticas para llevarlo a cabo así como criterios de evaluación claros y bien definidos. Pero también son conscientes de que no existen criterios para evaluar estos aspectos y que sería necesario tener normas de referencia para identificar los niveles de condición física.

Tabla 6. Perspectivas de los ATP sobre el estándar curricular tres para Educación Física en educación básica.

Estándar curricular 3. Evalúa los componentes de la condición física para mejorar el rendimiento físico y la salud.

Categorías

- Es importante por la problemática de sobrepeso y obesidad que presentan actualmente los niños.

- No existen estándares curriculares para la Educación Física en educación básica.

- Importante tener claro los criterios de evaluación.

- Importante para la salud de los niños.

- Los profes no evalúan la condición física.

- El programa y la política educativa no contemplan la evaluación de la condición física.

- Es importante el desarrollo de estrategias didácticas para trabajar este estándar.

- Estrategias didácticas creo que son necesarias.

- Criterios bien definidos para evaluar la condición física.

- Necesario conocer cómo se relaciona la condición física con la salud y que los niños entiendan.

- Necesario primero establecer las normas de referencias de los niveles de condición física.

- Que los estudiantes puedan conocer sus niveles de condición física.

- En los planes y programas no existen criterios para evaluar estos aspectos.

Nota: Elaborado por autores.

El grupo de los LEF (Ver Tabla 7) expone que es necesario más tiempo a la semana de Educación Física para educación básica, así como la importancia de los espacios y material didáctico, además perciben al estándar 1 como elemental y parte de las competencias que se esperan en esta asignatura.
Tabla 7. Perspectivas de los LEF sobre el estándar curricular uno para Educación Física en educación básica.

Estándar curricular 1. Demuestra el dominio de habilidades motoras y formas de movimiento necesarios para participar en una gran variedad de actividades físicas.

Categorías

- Deberían asignarse más horas en Educación Física.

- Se puede mejorar más si tuviéramos más clases por semana.

- Creo que sí es algo básico.

- Son importantes los espacios y el material didáctico.

- Sí lo trabajamos y es una de las competencias que se esperan en Educación Física.

- Es elemental en la Educación Física.

- $\quad$ Puede contribuir más claramente al desarrollo de las habilidades psicomotrices, ubicación del espacio y tiempo en los niños.

- $\quad$ Es parte de las competencias, de la manifestación global de la corporeidad, desarrollo de las habilidades y destrezas psicomotrices.

Nota: Elaborado por autores.

Con respecto al estándar 2 los LEF (Ver Tabla 8), indican que es difícil enseñar principios y conceptos, por el tiempo limitado en esta asignatura, pero que este estándar puede contribuir a la transversalidad, aunque los padres de familia y estudiantes no están familiarizados con conceptos, principios y estrategias.

Tabla 8. Perspectivas de los LEF sobre el estándar curricular dos para Educación Física en educación básica.

Estándar curricular 2. Demuestra y aplica conocimientos de conceptos, principios y estrategias de movimiento, necesarios para el aprendizaje de las habilidades motoras.

Categorías

- Es importante que aumenten las clases de Educación Física.

- Es difícil enseñar conceptos y principios.

- Son necesarias más clases de Educación Física.

- Es necesaria una evaluación escrita para este estándar.

- Que los padres de familia sepan que pueden reprobar o sacar bajas calificaciones en Educación Física.

- Puede ayudarnos a cumplir con la transversalidad.

- Sería difícil trabajar este estándar.

- Los niños y los padres de familia no están acostumbrados a ver conceptos, principios o estrategias en Educación Física.

- Es difícil alcanzar a ver este tipo de estándar una vez por semana.

Nota: Elaborado por autores. 
Tabla 9. Perspectivas de los LEF sobre el estándar curricular tres para Educación Física en educación básica.

Estándar curricular 3. Evalúa los componentes de la condición física para mejorar el rendimiento físico y la salud.

Categorías

- $\quad$ No existe en el programa la evaluación de la condición física.

- Sería importante para combatir el sobrepeso y la obesidad.

- $\quad$ Es importante que jueguen y se diviertan los niños.

- Creemos que es también importante la condición física y la salud.

- Creemos que si se divierten y mejoran la salud estaría bien.

- El programa no marca nada sobre la evaluación de la condición física.

- El programa actual se enfoca más en la corporeidad, juegos recreativos y psicomotricidad.

- Sería importante como parte de la activación física.

Nota: Elaborado por autores.

Los PIAF conciben al estándar 1 (Ver Tabla 10) como importante, sin embargo, también perciben que los maestros de Educación Física sólo ponen juegos y deportes, además de que existe poca inclusión de los niños con menos habilidades.

Tabla 10. Perspectivas de los PIAF sobre el estándar curricular uno para Educación Física en educación básica.

Estándar curricular 1. Demuestra el dominio de habilidades motoras y formas de movimiento necesarios para participar en una gran variedad de actividades físicas.

Categorías

- Los maestros de Educación Física solo ponen juegos y deportes.

- Deberían ser más formales y poner cosas como las que se manejan en el estándar.

- Este estándar puede contribuir a que los estudiantes sean personas activas físicamente.

- Es importante este estándar.

- Debe incluirse a todos los niños en las clases y que todos desarrollen esas habilidades.

- $\quad$ El profe sólo hace equipos representativos.

- No incluye a los niños con menos habilidades.

Nota: Elaborado por autores.

Las perspectivas de los PIAF del estándar curricular 2, (Ver Tabla 11) nos indican que existen deficiencias en la formación del educador físico y sería difícil implementar este tipo de estándares curriculares por el pobre nivel académico de los mismos.

Tabla 11. Perspectivas de los PIAF sobre el estándar curricular dos para Educación Física en educación básica.

Estándar curricular 2. Demuestra y aplica conocimientos de conceptos, principios y estrategias de movimiento, necesarios para el aprendizaje de las habilidades motoras.

Categorías

- Creo que es importante este estándar.

- Existe mucha deficiencia en el conocimiento de conceptos y principios morfológicos y fisiológicos en el profesional.

- Sería difícil para el licenciado en Educación Física llevarlo a cabo.

- Es muy deficiente la formación de licenciado en Educación Física en cuanto a principios y conceptos.

- Proporcionaría conocimientos reales y prácticos a los estudiantes para mejorar sus habilidades físicas y salud.

- Es difícil por el bajo nivel académico de los licenciados en Educación Física.

Nota: Elaborado por autores.

En relación con las perspectivas sobre el estándar 3, los PIAF (Ver Tabla 12) como muy poco recreativo a este estándar, debería replantearse mejor, pero podría ayudar a los niños a que sean más sanos y con buena condición física. Sería necesario preparar mejor a los estudiantes de la licenciatura en Educación Física y capacitar a los docentes de Educación Física en servicio, lo cual podría mejorar la calidad en el servicio de esta asignatura.

Tabla 12. Perspectivas de los PIAF sobre el estándar curricular tres para Educación Física en educación básica.

Estándar curricular 3. Evalúa los componentes de la Condición física para mejorar el rendimiento físico y la salud.

Categorías

- Es muy poco recreativo.

- Debería planearse bien.

- Replantear este estándar.

- Los niños y adolescentes serían más sanos con buena condición física.

- Poco recreativo.

- Preparar mejor a los estudiantes de la licenciatura.

- Capacitar a los que están en servicio.

- Puede ser importante para mejorar la calidad de la Educación Física.

- Puede mejorar su salud y mejorar su condición física.

Nota: Elaborado por autores. 


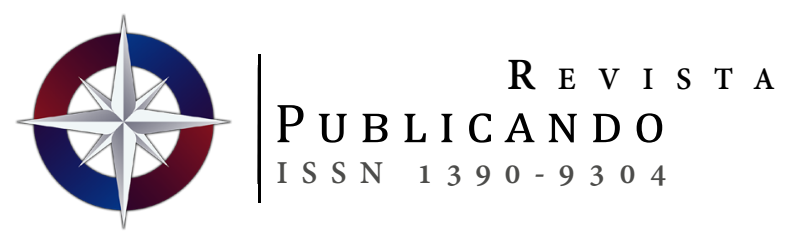

En general las categorías nos indican que los estándares curriculares para la Educación Física son importantes, pero también es necesario desarrollar algunas estrategias y romper ciertos paradigmas para poder implementarlos en la clase de Educación Física en educación básica.

\section{CONCLUSIONES Y DISCUSIONES}

En el estándar 1, el estudiante debe "Demostrar el dominio de habilidades motoras y formas de movimiento necesarios para participar en una gran variedad de actividades físicas", este estándar es concebido por los GF como importante y elemental para la Educación Física, sin embargo, también perciben que las horas por semana de esta asignatura en México pueden ser insuficientes para cumplir con este estándar. Esta postura es consistente con las políticas en materia de Educación Física, en donde se recomiendan de 120 a 180 minutos por semana para primaria según la CDC (Centers for Disease Control and Prevention, 2014) y la UNESCO, 2015. En México sólo se contemplan de 50 a 100 minutos semanales, por lo que sería necesario aumentar minutos en las sesiones de Educación Física, para implementar estándares curriculares en esta asignatura. Si a esto le sumamos las evidencias de un estudio hecho en las clases de Educación Física en escolares mexicanos, en el cual concluyen que la actividad física en el recreo, es mayor que la misma que se realiza en la clases de esta asignatura (Hall-López, Ochoa-Martínez, Monreal y Sáenz-López, 2017), podemos suponer que el programa curricular necesita mejorar en estos aspectos, además del tiempo destinado a la Educación Física.

Por otra parte, el estándar uno articula claramente las habilidades físicas y motrices, como correr, trotar, saltar, empujar, lanzar, golpear, para que el estudiante pueda participar en diferentes actividades físicas, por lo que es consistente con la alfabetización física para el desarrollo de estilos de vida activos y saludables (Longmuir et al., 2015; UNESCO, 2015). Estas perspectivas nos indican que es fundamental el trabajo de este estándar curricular en los estudiantes de educación básica, pero, también se percibe una necesidad de aumentar el tiempo destinado a la Educación Física en educación básica en primaria.

Existen estudios que concluyen que las habilidades motoras y la capacidad cardiovascular en niños tienen una fuerte correlación con la actividad física que realizan diariamente y que la Educación Física tiene un impacto positivo sobre estas dos variables (CDC, 2014; Larouche, Boyer, Tremblay y Longmuir, 2013). También existe evidencia de que las habilidades motoras trabajadas en la clase de Educación Física contribuyen positivamente en los logros académicos, mejorando las habilidades cognitivas y de interacción social (Ericsson y Karlsson, 2014; Leonetti, Zhu y Chen, 2016). Por lo que el estándar uno, cumpliría con el desarrollo de habilidades motoras y formas de movimiento necesarias para que los escolares realicen actividades físicas, lo que fundamenta la percepción de los GF hacia este estándar curricular como importante y necesario para la Educación Física en educación básica en primaria, sin embargo, la falta de criterios de evaluación es consistente con un estudio hecho por Dyson et al. (2011), en el cual concluye que es necesario diseñar y validar más instrumentos para evaluar este tipo de estándares curriculares en la Educación Física.

En cuanto al estándar 2, el estudiante debe "Demostrar y aplicar conocimientos de conceptos, principios y estrategias de movimiento, necesarios para el aprendizaje de las habilidades motoras" este es considerado importante en el desarrollo cognitivo y procedimental, los GF lo conciben como una oportunidad para mejorar la calidad académica de la Educación Física. En México la SEP establece un modelo por competencias y estándares que involucra lo procedimental, conceptual y actitudinal basado en el modelo PISA (SEP, 2010; SEP, 2011), por lo que este estándar es consistente con el modelo educativo en México y el perfil de egreso de la educación básica, al brindar al estudiante un dominio académico de conocimientos, conceptos, principios y estrategias relacionados con las habilidades motrices para la vida.

Para implementar el estándar 2, los GF consideran necesario mejorar la formación profesional del Educador Físico, así como capacitar y certificar a los docentes de Educación Física en servicio, esta percepción sobre el Educador Físico es contrastante con un estudio hecho por Cárcamo en el 2012. En dicho estudio, se encuestan a escolares de $5^{\circ} \mathrm{y}$ $6^{\circ}$ de educación básica y de $1^{\circ}$ y $2^{\circ}$ de educación media, los estudiantes perciben a los Educadores Físicos como competentes, preparados y seguros, además de permitir generar actitudes positivas hacia la Educación Física y así crear el hábito de realizar actividad física. La realidad es que aún existen retos y es necesario romper paradigmas y mejorar la formación profesional del educador físico, dotarlo de conocimientos, habilidades y actitudes necesarias para brindar un servicio de calidad en la Educación Física. Es necesario que el educador físico logre que los estudiantes de educación básica desarrollen estilos de vida activos y saludables utilizando diferentes disciplinas, como la Fisiología del ejercicio, Biomecánica, Kinesiología, Sociología del deporte entre otras (Jin, 2013; Veloo y Md-Ali, 2016).

El AAHPERD (American Alliance for Health, Physical Education, Recreation and Dance, 2013) y el ACARA (Australian Curriculum, Assessment and Reporting Authority, 2012) son modelos educativos en donde se expresan estándares relacionados con la aplicación de conocimientos de principios, conceptos y estrategias, en estos estándares curriculares el estudiante debe "explicar, describir y analizar 


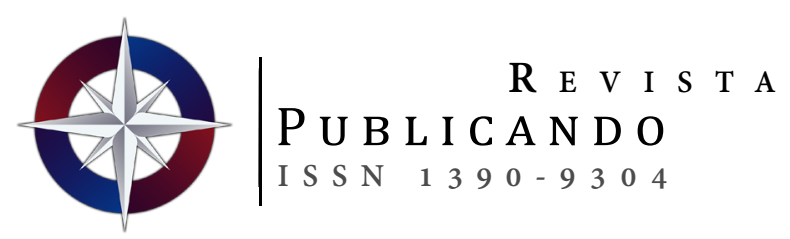

conceptos relacionados con la condición física y la salud" o "Demostrar comprensión de conocimientos de conceptos, principios y estrategias aplicadas al aprendizaje y demostración de actividades físicas". Además, este tipo de estándares curriculares contribuye a que el estudiante desarrolle conocimientos y lo lleva a la aplicación para la vida, por lo que se vuelve necesario este estándar y además también converge con las demandas y políticas globales de Educación Física de calidad (CDC, 2014).

En el estándar 3, el estudiante debe aprender a "evaluar los componentes de la condición física para mejorar el rendimiento físico y la salud", en este caso los GF resaltan que la Educación Física en México no contempla criterios ni normas de referencia para evaluar la condición física relacionada con la salud de los estudiantes. Aún más importante la relevancia de tener criterios para evaluar la condición física y sobre todo que se identifiquen curricularmente los logros de los estudiantes son sumamente importantes, puesto que existe evidencia que vincula la obesidad con una pobre condición física en escolares (Gálvez et al., 2015; Ruíz et al., 2011). El estándar 3 ofrece a los estudiantes los conocimientos y habilidades necesarias para poder medir, evaluar e identificar los indicadores o parámetros para desarrollar y mantener una condición física saludable a lo largo de la vida, lo cual es convergente con el rol de la Educación Física en la política pública (CDC, 2014; Sallis, McKenzie, Beets, Beighle, Erwin y Lee, 2012; Veloo y Md-Ali, 2016). Si tomamos en cuenta que en algunos países ya existen pruebas físicas estandarizadas y validadas para la población infantil (Ruíz et al., 2011), es posible considerar que en el caso de México y Latino América existe un atraso en la evaluación de la condición física relacionada con la salud, por lo que sería necesario validar y estandarizar pruebas físicas en nuestra población para poder trabajar o implementar este estándar curricular en Educación Física.

Los GF perciben que no existe mucha consistencia entre la condición física relacionada con la salud en México, al ofertar más actividades que involucran la corporeidad, expresión corporal, juegos recreativos, deportes, teatro y psicomotricidad en el currículo de Educación Física (SEP, 2011; SEP, 2017). Sin embargo, las problemáticas de sobrepeso y obesidad infantil que se presentan actualmente en México según los datos presentados en la encuesta de medio camino realizada por el INSP (Instituto Nacional de Salud Pública, 2016), deberían sustentar este tipo de estándar como una ayuda a combatir las problemáticas antes mencionadas. Lo anterior pone de manifiesto la necesidad de promover políticas y reformas curriculares que permitan el desarrollo de conocimientos de conceptos, principios y estrategias vinculadas con la mejora de la condición física relacionada con la salud de los escolares, desde la clase de Educación Física en México y posiblemente en Latino América (García y Del Basto, 2017; Starc y Strel, 2012). En este sentido podemos inferir que la parte epistemológica y hermenéutica de la Educación Física que busca mejorar y mantener los componentes de la condición física saludable, así como promover la actividad física para la salud, parecen estar un algo ausente en el currículo.

En general se puede concluir que las perspectivas de los GF, nos indican que los estándares curriculares pudieran mejorar la calidad en el servicio de la Educación Física, sin embargo, también se concluye que se requiere capacitar y actualizar a los docentes en servicio para implementarlos. Además, existe una concepción en la cual se debe mejorar la formación profesional del LEF para el trabajo con estándares curriculares, así como una necesidad de diseñar aprendizajes esperados, estrategias didácticas y criterios de evaluación bien definidos para llevar a cabo la implementación de estos.

Sin duda el trabajo con estándares curriculares, según los GF, puede ser una oportunidad para mejorar la Educación Física en educación básica, pero se requiere de un esfuerzo conjunto para romper ciertos paradigmas entre los diferentes sectores educativos y de la sociedad, además de crear políticas educativas y de salud pública para fortalecer esta asignatura y a los educadores físicos. 


\section{REFERENCIAS}

American Alliance for Health, Physical Education, Recreation and Dance (AAHPERD), (2013). Grade-level outcomes for K-12 physical education, en: <https:// www.shapeamerica.org/standards/default.aspx $>$. [Consulta: 25-03-2018]

Australian Curriculum, Assessment and Reporting Authority (ACARA), (2012). La Forma del Currículo de Australia: Educación Física y Salud, en: <https:// docs.acara.edu.au/resources/Shape_of_the_Australian_Curriculum_Health_and_Physical_Education.pdf $>$. [Consulta: 25-01-2017]

Cárcamo, J. (2012). El profesor de Educación Física desde la perspectiva de los escolares. Revista Estudios Pedagógicos, (1), 105-119, en: <http://revistas.uach.cl/ index.php/estped/article/view/2746>

CDC. (2014). Physical Education Profiles, 2012: Physical Education and Physical Activity Practices and Policies Among Secondary Schools at Select US Sites. Atlanta, GA: Centers for Disease Control and Prevention, US Dept of Health and Human Services. [Consulta: 17-05-2019], en: <https://www.cdc.gov/ healthyschools/physicalactivity/facts.htm >

Dyson, D., Placek, J. H., Graber, H. C., Fisette, J. L., Rink, J., Zhu, W., Avery, M., Franck, M., Fox, C., Raynes, D. \& Park, Y. (2011). Development of PE Metrics Elementary Assessments for National Physical Education Standard 1. Measurement in Physical Education and Exercise Science, 15:2, 100-118, DOI: 10.1080/1091367X.2011.568364

Ericsson, I. y Karlsson, M. K. (2014). Motor skills and school performance in children with daily physical education in school - a 9-year intervention study. Scand J Med Sci Sports, 24: 273-278. DOI: 10.1111/j.16000838.2012.01458.x

Gallo, L, E. y Urrego, L., (2015). Estado del Conocimiento de la Educación Física en la Investigación Educativa. Perfiles Educativos, vol.37, n.150, pp.143-155.

Gálvez, C., Rodríguez, P. L., Rosa A., García-Cantó, Eliseo., Pérez, J. J., Tárraga, M. L., \& Tárraga, P. J. (2015). Nivel de condición física y su relación con el estatus de peso corporal en escolares. Nutrición Hospitalaria, 31(1), 393-400. https://dx.doi.org/10.3305/ nh.2015.31.1.8074
García, J, M. y Del Basto, L, M., (2017). Revisión Internacional e Iberoamericana de Currículum de Educación Física. El caso de México. Revista Iberoamericana sobre Calidad, Eficacia y Cambio en Educación, vol. 15, núm. 1, 2017, pp. 71-8515 (1), 71-85.

Hall-López, J. A., Ochoa-Martínez, P. Y., Zuñiga, R., Monreal, L. R. y Sáenz-López, P. (2017). Actividad física moderada a vigorosa durante el recreo y clase de Educación Física en niños mexicanos de escuela primaria. Retos, 31, 137-139, en: <https://recyt. fecyt.es/index.php/retos/article/view/49640/32135 >. [Consulta: 27-01-2020.

Hamui-Sutton, A. y Varela-Ruíz, M. (2013). La técnica de grupos focales, Inv Ed Med educación médica, 2 (1): 55-60, en: <http://www.scielo.org.mx/scielo. php?pid=S2007-50572013000100009\&script=sci_abstract $>$. [Consulta: 21-05-2015].

Instituto Nacional de Salud Pública (INSP). (2016). Encuesta Nacional de Salud y Nutrición de medio camino, 2016, [Consulta: 25-11-2018] en: <https://ensanut. insp.mx/encuestas/ensanut2016/index.php >.

International Council for Health, Physical Education, Recreation, Sport, and Dance (ICHPER•SD). (2009). [Consulta: 18-11-2016], en: <http://www.ichpersd. org/index.php/standards/international-standards $>$

Jin, A. (2013). Physical education curriculum reform in China: a perspective from physical education teachers. Physical Education \& Sport Pedagogy, 18(1), 15-27. doi:10.1080/17408989.2011.623231

Larouche, R., Boyer, C., Tremblay, M. S. y Longmuir, P. (2013). Physical fitness, motor skill, and physical activity relationships in grade 4 to 6 children. Physiol. Nutr. Metab., 39: 553-559. dx.doi.org/10.1139/ apnm-2013-0371.

Leonetti, M., Zhu, X. y Chen, S. (2016). Improving students' knowledge and values in physical education through Physical Best lessons. European Physical Education Review, Vol 23, No. 2, 223 - 236. https://doi. org/10.1177/1356336X16645366

Liang, G., Walls, R. T. y Lu, C., (2005). Standards and practice for physical education in China. JOPERD, 76, (6).

Longmuir, P. E., Boyer, C., Lloyd, M., Yang, Y., Boiarskaia, 


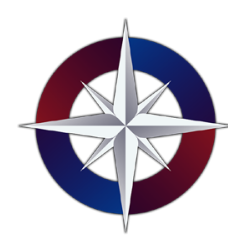

E., Zhu, W. and Tremblay, M. S. E., Boyer, C., Lloyd, M., Yang, Y., Boiarskaia, E., Zhu, W. and Tremblay, M. S.(2015). The Canadian Assessment of Physical Literacy: methods for children in grades 4 to 6 (8 to 12 years). BMC Public Health 15:767. DOI 10.1186/s12889-015-2106-6

Lound, J. y Tannehill, D. (2014). Introduction to Standards-Based Curriculum Development, en: <http://samples.jbpub. com/9781284034196/9781449691745_CH01_ SAMP.pdf>. [Consulta: 12-12-2016]

Marani, L., Sanches Neto, L. y dos Santos Freire E. (2017). El currículo de la Educación Física en la red municipal de Barueri: Percepción de los docentes. Revista de movimiento, 23, (1). 249-264.

Melnychuk, N., Robinson, D. B., Lu, C., Chorney, D. y Randall, L. (2011). Physical Education Teacher Education in Canada. Canadian Journal of Education, 34, (2). $148-168$.

Organización de las Naciones Unidas para la Educación, la Ciencia y la Cultura (UNESCO). (2015). Educación Física de Calidad: Guía para responsable políticos. 2015. [Consulta: 30-09-2017], en: <http://unesdoc. unesco.org/images/0023/002313/231340s.pdf>

Programme for International Student Assessment "PISA". (2006). Science Competencies for Tomorrow's World. Vol. 1. OECD.

Reyes, A., Rivas, J. y Pávez-Adasme, G. (2020) Tiempo de compromiso motor en la clase de Educación Física, Voces De La Educación, 5(10), p. 90-113.

Ruiz, J. R., España, V., Castro, J., Artero, E. G., Ortega F. B., Cuenca, M., Jiménez Pavón D., Chillón P., Girela Rejón M. J., Mora J, Gutiérrez A., Suni J., Sjöstrom M., Castillo Mj (2011). Batería ALPHA-Fitness: test de campo para la evaluación de la condición física relacionada con la salud en niños y adolescentes, Nutrición Hospitalaria, 26 (6): 1210-1214.

Sallis, J. F., McKenzie, T. L., Beets, M. W., Beighle, A., Erwin, H. y Lee, S. (2012). Physical Education's Role in Public Health: Steps Forward and Backward Over 20 Years and HOPE for the Future. AAHPERD, 83 (2): 125-135.

Secretaria de Educación Pública (SEP) (2017), Aprendizajes Clave, Educación Física. (n.d.). 160-187. en: $<$ https://www.planyprogramasdestudio.sep.gob. $\mathrm{mx} /$ descargables/biblioteca/basica-educ-fisica/1LpMEducacion-Fisica_Digital.pdf $>$. [Consulta: 3011-2019]

SEP (2010). Estándares Curriculares para la Educación básica En México. Versión preliminar. Subsecretaría de Educación Básica, en: <https://ocadizquintanar. files.wordpress.com/2011/06/estandares-curriculares.pdf $>$. [Consulta: 18-09-2017]

SEP (2011). Plan de estudios de educación básica de la SEP, en:<http://basica.sep.gob.mx/dgdc/sitio/pdf/PlanEdu2011.pdf $>$. [Consulta: 20-09-2017]

Society of health and Physical Educators (SHAPE). (2015). The Essential Components of Physical Education, en: <https://www.shapeamerica.org/publications/ resources/teachingtools/teachertoolbox/Teachers_Toolbox_Elementary_PE.aspx $>$. [Consulta: 05-11-2019]

Starc, G. y Strel, J. (2012). Influence of the quality implementation of a physical education curriculum on the physical development and physical fitness of children. BMC Public Health, 12:61. Disponible en: http://www.biomedcentral.com/1471-2458/12/61 doi:10.1186/1471-2458-12-61.

Veloo, A. y MD-Ali, R. (2016). Physical Education Teachers Challenges in Implementing School Based Assessment. International Review of Management and Marketing, 6 (S8) 48-53. 